Caries Res. 1969;3:377-380

\title{
Subject Index Vol. 3
}

Abrasiveness of fluoride prophylaxis pastes,

225 Albino ferret dentition, 369 -, usefulness for caries research, 212 Aluminium factories, environmental effect

on caries, 221 Amalgam fillings, and in vitro caries, 213 Ammonium effect on streptococcal fermentation of sucrose, 226 Amylase distribution in salivary glands, 210 Apatite, incorporation of hydroxyl and

fluoride, 219 Arylaminopeptidases in normal and carious dentine, 134

Bacteria, polysaccharide-producing, and

fluoride in water, 178 Bone meal, cariostatic effect in the hamster,

215

Calcifying fluids, influence on in vitro

enamel lesions, 214 Calcium in human plasma and saliva, 211 Carbohydrate breakdown in plaque, 249 Caries in albino rats, and sucrose and

starch, 208

in animals, and trace elements, 1

in an animal experiment, 200

in children, and streptococcal poly-saccharide, 190

in children, and water fluoride, 178

-, possible demineralization by complex

forming agents, 213 -, and deciduous enamel structure, 218

- $\quad$ in enamel, effect of fluoride rinsings, 226

-, enhanced by selenium, 14 -, environmental effect of aluminium factories, 221

- $\quad$ etiology, New Guinea study, 44

-, experimental in man, 206

-, geographic variation in USA, 32

- $\quad$ in the hamster, effect of dextrans, 205

increment after withdrawal of fluoride dentifrice, 226

induction in monkeys, 227

inhibiting effect of hexafluorostannate, 315

inhibition in rats by fluoride preparations, 225

inhibition in dentine by hexafluorostannate, 224

prevalence in Uganda, 222

prophylaxis by fluoride, experimental and clinical tests, 281

-, rampant, induction in monkeys, 207

- $\quad$ in the rat, trimetaphosphate effect, 326

-, relation to water fluoride, 223

- $\quad$ resistant recruits, mineral environment,

23

-, response of deciduous dentine, 348 -, response of dentine, 217 
around silver amalgam fillings, 213

in young diabetics, 215

Cariogenic streptococcus, sucrose fermentation influenced by K, Na, N3/4, 266

Cariostatic effect of bone meal in the hamster, 215

Clinical trial of hexafluorostannate, 315

378

Subject Index Vol. 3

Complex forming agents, possible role in

carious demineralization, 213 Crystallography, sampling technique of

dental hard tissues, 216 Cystine cleaving enzymes in normal and

carious dentine, 134

Deciduous dentine, response to caries, 348 Demineralization, in caries, possible role of

complex forming agents, 213 Density of human parotid fluid, 149 Dental conditions, relationship

to molybdenum, 75

- $\quad$ hard tissues, sampling technique for

electron microscopy and crystallogra

phy, 216

- $\quad$ tissues, content of trace elements, 60

Dentine, deciduous, response to caries, 348

-, caries inhibition by hexafluorostannate,

224 -, carious, phosphorolytic capacity, 210 -, normal and carious, enzyme content, 134 -,

response to caries, 217 Dentition of the albino ferret, 369

- $\quad$ of diabetics, 215

Dextrans, effect on hamster caries, 205

Diabetics, caries, 215

-, dentition, 215

Dissolution of enamel and fluorapatite, 220

Electron microscopy, sampling technique

on dental hard tissues, 216 -, scanning, of pretreated enamel, 225 Enamel biopsy for determining in vivo

fluoride uptake, 119

- $\quad$ caries, effect of fluoride rinsings, 226

-, deciduous, relationship to dental disease, 218

-, demineralization studies by scanning electron microscopy, 225

- $\quad$ dissolution studies, 220

fluoride concentration and caries susceptibility, 223

lesions in vitro, histopathology, 214

-, microdistribution, uptake and loss of fluoride, 109

- $\quad$ protein, embryonic, gel filtration, 217

-, variations in composition, 221

Enzymes, in normal and carious dentine,

134

Fermentation of sucrose, streptococcal, effect of K, Na, NH4, 266

Ferret, albino, usefulness for caries research, 212

- $\quad$ dentition, 369 
Fluorapatite, dissolution studies, 220

-, tracer exchange studies, 220

$18 \mathrm{~F}$, penetration to the pulp from solutions and fillings, 214

Fluoridated water, caries and poly-saccharide-producing bacteria, 178

Fluoridation of salt, two year clinical results, 222

Fluoride analysis of soft dental deposits, 224

concentration in enamel and caries susceptibility, 223

dentifrice, effect on caries of withdrawal, 226

dentifrices, $\mathrm{F}$ retention in preschool children, 290

dosage with salt, urinary estimation, 218

in drinking water, relation to caries and fluorosis, 223

electrode for urinary estimation of F intake, 218

-, excretion in human saliva and milk, 159 -, incorporation in apatite lattice, 219

ingestion and excretion of monofluoro-phosphate, 219

intake, estimation in urine by F electrode, 218

-, in vivo uptake determined by enamel

biopsy, 119 -, microdistribution, uptake and loss in

enamel, 109

mouth rinses containing manganese, 99

mouth rinses, F retention in preschool children, 290

preparations inhibiting rat caries, 225

pretreatment of enamel, 225

prophylaxis of caries, experimental and clinical tests, 281

prophylaxis pastes, abrasiveness and $\mathrm{F}$ uptake, 225

retained from mouth rinses and dentifrices, 290

rinsings, influence on enamel caries, 226

- $\quad$ uptake from prophylaxis pastes, 225

Fluorine, position as impurity in hydro-

xylapatite, 222 Fluorosis, dental, prevalence in Uganda,

222 -, relation to water fluoride, 223

Subject Index Vol. 3

379

Follicles of unerupted teeth, 216

Gel filtration of embryonic enamel protein,

217 Geographic variation of caries in USA, 32 Glucose, catabolism by a caries-conducive

streptococcus, 167 -, in human plasma and saliva, 211 Glycoprotein, sublingual, properties, 211 , virus haemagglutination-inhibiting, distribution in salivary glands, 210

Hamster caries, effect of dextrans, 205 -, cariostatic effect of dietary bonemeal,215

Hexafluorostannate, clinical caries-inhibition, 315 -, inhibiting dentinal caries, 224

Hydroxylapatite, adsorption of sublingual

glycoprotein, 211 -, position of fluorine as impurity, 222 Hypoxia, experimental influence on the rat incisor, 308

Induction of caries in monkeys, 227 Iodophilic polysaccharide-producing bacteria, caries and fluoridated water, 178 Ionic influence on streptococcal fermentation, 208

Lysozyme, distribution in salivary glands, 210 
Macaca irus, induction of caries, 227 -, induction of rampant caries, 207 Manganese in fluoride mouth rinses, 99 Matrix polysaccharies in dental plaque, 206 Microbial changes by

trimetaphosphate in

the rat, 326 Microscopy, light and electron, of caries in

deciduous dentine, 348 Milk, human, fluoride content, 159 Mineral environment of caries

resistant

recruits, 23 Mineralisation defects, following neonatal

asphyxia, 301 Mitis-salivarius agar for differentiating

plaque streptococci, 207 Molybdenum and dental conditions, 75 Monkey caries induction, 227 plaque, polysaccharide formation, 205 Monkeys, induction of rampant caries, 207

Monofluorophosphate, ingestion and excretion in children, 219 Mouth rinses with fluoride

solution containing manganese, 99

Neonatal asphyxia and mineralisation defects, 301

Nutrition and caries etiology in New Guinea, 44

in a blackhooded rat strain, 200

ORCA Congress 1969, abstracts, 205 Osmolality of human parotid fluid, 149

32P, penetration to the pulp from solutions

and fillings, 214 Parotid fluid, density and osmolality, 149 Penetration to the pulp of 18F and

32P from

solutions and fillings, 214 Perinatal hypoxia, influence on the rat

incisor, 308 Phosphate in human plasma and saliva, 211 Phosphatases in normal and carious

dentine, 134

- $\quad$ in plaque, effect of trace elements, 209

Phosphorolysis of plaque, effect of trace

elements, 209 Phosphorolytic capacity of plaque, saliva

and carious dentine, 210 Phytin, effect on rat plasma, saliva, bone

and teeth, 212 Plaque, content of trace element, 60 -, fluoride analysis, 224 -, matrix

polysaccharides, 206 -, in monkey, polysaccharide formation,

205 -, phosphorolytic capacity, 210 -, distribution and characteristics, 238

- $\quad$ streptococci, differentiated on mitis-

salivarius agar, 207

Plasma, contents of glucose, calcium and

phosphate, 211 Polysaccharide, bacterial, and fluoridated

water, 178 -, streptococcal, and caries in children, 190 Polysaccharides, formation in monkey

plaque, 205

- $\quad$ of plaque matrix, 206

Potassium effect on streptococcal fermentation of sucrose, 266

Primary teeth, mineralisation defects following neonatal asphyxia, 301

Prophylaxis of caries by fluoride, experimental and clinical tests, 281

- $\quad$ pastes, abrasiveness and fluoride uptake,

225

380

Subject Index Vol. 3

Proteases, from oral streptococci, purification, 209 Protein breakdown in plaque, 249 - matrix of plaque, breakdown, 249 
Rat caries, inhibited by fluoride preparations, 225

Rats, black-hooded, in a caries experiment, 200

Response of deciduous dentine to caries, 348

Retention of fluoride from mouth rinses and dentifrices, 290

Saliva, contents of glucose, calcium and phosphate, 211

-, human, fluoride content, 159

-, phosphorolytic capacity, 210

Salivary glands, distribution of alpha-amylase, lysozyme and virus haemagglu-tination-inhibiting glycoprotein, 210

Salt fluoridation, two year clinical results, 222

-, urinary estimation of dosage, 218

Scanning electron microscopy of pretreated enamel, 225

Selenium, a caries enhancing element, 14

Silicate cement, penetration to the pulp of $18 \mathrm{~F}$ and 32P labels, 214

Sodium effect on streptococcal fermentation of sucrose, 266

Stannous fluoride, water-free, for topical application, 339

Starch and caries in albino rats, 208

Streptococcal fermentation of sucrose, ionic influences, 208

Streptococci, distribution and characteristics in plaque, 238

-, of human plaque, differentiation, 207

-, polysaccharide-producing, and caries in children, 190

Streptococcus, caries-conducive, catabo-lism of glucose, 167

-, cariogenic, sucrose fermentation influenced by K, Na, NH4, 266

- $\quad$ zymogenes proteases, purification, 209

Sublingual glycoprotein, properties, 211

Sucrose and caries in albino rats, 208

fermentation, streptococcal, influence of K, Na, NH4, 208

fermentation, streptococcal, effect of K, Na, NH4, 266

Toothbrushing with fluoride dentifrice, effect on caries of withdrawal, 226

Topical application using water-free stannous fluoride, 339

Trace elements and animal caries, 1

and caries in New Guinea, 44

of dental tissues and plaque, 60 -, effect on plaque phosphatases, 209 -, round table discussion,

88 Tracer studies on fluorapatite, 220 Trimetaphosphate influence on rat caries

and microbiota, 326

Uganda, prevalence of dental caries and

fluorosis, 222 Unerupted teeth and their follicles, 216 Urinary estimation of fluoride dosage with salt, 218

- $\quad$ F excretion, estimation of intake by

F electrode, 218

Water fluoride, relation to caries and

fluorosis, 223 Water-free stannous fluoride for topical

application, 339 